streitgegenständliche Teppich aus dem Teppichhaus S. stammte, und deswegen keinen dahingehenden Herkunftshinweis aufgenommen.

Auch wenn die Klagepartei in ihrem schriftsätzlichen Sachvortrag immer wieder einen entsprechenden Bezug hergestellt hat, kann diese Herkunft mangels eines entsprechenden Etiketts nicht sicher festgestellt werden.

Soweit der Zeuge H. in seiner Aussage vom 20. Februar 2014 zunächst die Provenienz des Teppichs aus der Sammlung S. bestätigt hatte, räumte er auf Nachfrage ein, das er diese Erkenntnis nur von einem Bekannten habe, der ihm aus Presseveröffentlichungen zu diesem Rechtsstreit berichtet hatte.

Die frühere Haushälterin des Teppichexperten S. hat nach dem klägerischen Sachvortrag auf S. 6 des Schriftsatzes vom 26. November 2010 nicht unmittelbar Herrn S. beerbt, sondern den zweiten Ehemann der Witwe S., Herrn P., der seinerseits Antiquitäten gesammelt hat. Der Teppich könnte daher auch aus der originären Sammlung von Herrn P. stammen.

Da die Beklagte nur die Angaben zur Beschreibung des Teppichs in ihren Katalog aufnehmen durfte, die verlässlich sind, kann ihr unter diesen Umständen nicht vorgeworfen werden, dass sie in Bezug auf den streitgegenständlichen Teppich keinen Hinweis auf eine Herkunft aus dem Teppichhaus S. aufgenommen hat.

Im Übrigen hat der von der Beklagten versteigerte weitere Teppich, bei dessen Katalogbeschreibung ausdrücklich auf die Herkunft aus dem Teppichhaus S. hingewiesen worden war, ausweislich der Abrechnung einen Versteigerungserlös von 2.700 Euro erzielt, also wie der streitgegenständliche Teppich einen Erlös ca. in Höhe des 22 fachen Katalogpreises.
3. Schließlich trifft die Beklagte auch nicht der Vorwurf des Übernahmeverschuldens oder einer Verletzung einer Hinweisbzw. Beratungspflicht.

Wie oben unter 2.3. ausgeführt wurde, war es für die Beklagte nicht erkennbar, dass es sich bei dem - neben zahlreichen weniger wertvollen Gegenständen - aus dem präsentierten Nachlass übernommenen persischen Teppich um ein besonders wertvolles Exemplar aus der Zeit vor dem 19. Jahrhundert handelte.

Insoweit bestand für die Beklagte keine Veranlassung, die Klagepartei an ein überregionales Auktionshaus zu verweisen.

Die Beklagte musste die Versteigerung des Teppichs auch nicht bereits deshalb ablehnen, weil ihr eine genauere Zuordnung als vorgenommen nicht möglich war.

Gerade bei Nachlassgegenständen ist nach der Lebenserfahrung die genaue Herkunft häufiger gar nicht oder nicht mit zumutbarem Aufwand klärbar.

Wie oben dargestellt, war die Katalogbeschreibung zutreffend. Dass diese nicht besonders präzise bzw. detailliert war, konnte auch die Klagepartei erkennen.

Insoweit war auch der von der Klagepartei verlangte ausdrückliche Hinweis, dass die Beklagte den Teppich nicht näher einordnen könne, nicht erforderlich.

4. Da es auf spezielle Fragen zu antiken Orientteppichen, für die der gerichtliche Sachverständige nicht als Gutachter bestellt ist, nicht entscheidungserheblich ankam, war die zusätzliche Einholung des Gutachtens eines Experten für Orientteppiche nicht erforderlich. (Entscheidung von der Redaktion bearbeitet.)

\title{
Strafbarkeit der Einfuhr von ägyptischen Kulturgütern
}

Landgericht Freiburg, Beschluss vom 12. Dezember $2013-8$ Qs 7/13

Dem Verletzten durch die Straftat entzogen im Sinne des §111k S. 1 StPO ist eine Sache auch dann, wenn nur ein Teilaspekt des Gesamtgeschehens nach deutschem Recht strafbar ist und die im Strafverfahren abgeurteilte Tat Teil eines einheitlichen Lebenssachverhalts im Sinne des § 264 StPO ist, der zur Entziehung der Sache geführt hat. (Amtlicher Leitsatz)

\section{Gründe}

I.

- Der Beschwerdeführer reiste am 13. Februar 2009 am Grenzübergang Weil am Rhein aus der Schweiz in die Bundesrepublik Deutschland ein. Im Fahrzeug führte er vier ägyptische Kulturgüter (1 Miniaturobelisk, 1 rechteckige Nischenstele, 1 Statu- enschrein und 1 Schnurösengefäß) mit, die er über die Grenze bringen wollte, ohne deren Einfuhr anzumelden. Hierdurch wollte er die Erhebung der Einfuhrumsatzsteuer in Höhe von mindestens 3.920 Euro umgehen. Bei der Grenzkontrolle wurden die Kulturgüter jedoch aufgefunden und gemäß §§ 94, 98 StPO als Beweismittel sichergestellt. 
Durch Strafbefehl des Amtsgerichts Lörrach vom 13. August 2012 wurde gegen den Beschwerdeführer wegen Steuerhinterziehung und versuchter Steuerhinterziehung eine Gesamtgeldstrafe in Höhe von 90 Tagessätzen zu jeweils 45 Euro festgesetzt. Der Strafbefehl ist seit dem 30. August 2012 rechtskräftig.

Auf Antrag der Staatsanwaltschaft Freiburg - Zweigstelle Lörrach - hat das Amtsgericht Lörrach mit Beschluss vom 11. Juni 2013 gemäß § 111k StPO die Herausgabe der noch sichergestellten vier Kunstgegenstände an die Arabische Republik Ägypten angeordnet.

Hiergegen wendet sich die mit Verteidigerschriftsatz vom 18. Juni 2013 eingelegte und mit Schriftsatz vom 9. August 2013 begründete Beschwerde.

Die zulässig eingelegte Beschwerde hat in der Sache nur einen geringen Teilerfolg.

\section{II.}

Das Amtsgericht Lörrach war auch nach rechtskräftigem Abschluss des Strafverfahrens auf Antrag der Staatsanwaltschaft Freiburg - Zweigstelle Lörrach - gemäß § 111k S. 3 StPO zur Entscheidung über die Herausgabe zuständig.

\section{III.}

Grundsätzlich sind beschlagnahmte Sachen, wenn sie für das Strafverfahren nicht mehr benötigt werden, an den letzten Gewahrsamsinhaber herauszugeben. Eine Ausnahme von diesem Grundsatz sieht $\S 111 \mathrm{k}$ StPO vor. Hiernach soll eine bewegliche Sache, die nach $\S 94$ beschlagnahmt oder sonst sichergestellt oder nach § 111c Abs. 1 beschlagnahmt worden ist und für Zwecke des Strafverfahrens nicht mehr benötigt wird, dem Verletzten, dem sie durch die Straftat entzogen worden ist, herausgegeben werden, wenn er bekannt ist und Ansprüche Dritter nicht entgegenstehen. Diese Voraussetzungen sind bezüglich der drei Gegenstände Miniaturobelisk, rechteckige Nischenstele und Statuenschrein für die Arabische Republik Ägypten als Verletzte erfüllt.

1. § $111 \mathrm{k}$ StPO ist vorliegend grundsätzlich anwendbar. Die Vorschriften des KulturgüterrückführungsG über die Rückgabepflicht und die Anhaltung von Kulturgut stellen gegenüber $\S 111 \mathrm{k}$ StPO keine lex specialis dar, das die Anwendung von $\S 111 \mathrm{k}$ StPO ausschlösse. Das KulturgüterrückführungsG regelt nämlich den Umgang mit Kulturgütern auch für den Fall, dass die Gegenstände nicht im Rahmen eines Strafverfahrens beschlagnahmt wurden.

2. Die Voraussetzungen des $\S 111 \mathrm{k}$ S. 1 StPO liegen im Hinblick auf die drei genannten Gegenstände auch vor. a) Die Gegenstände wurden im Rahmen des Strafverfahrens gegen den Verurteilten B. sowie die getrennt verfolgten J.B. und E.M. beschlagnahmt, als der Verurteilte B. mit ihnen aus der Schweiz in die Bundesrepublik Deutschland einreiste, ohne sie zu deklarieren. Sie werden für das Strafverfahren nicht mehr benötigt.

b) Die Arabische Republik Ägypten ist bezüglich der drei genannten Gegenstände Verletzte.

Gemäß Art. 1 des ägyptischen Antikengesetzes gelten bewegliche Gegenstände als „Antike“, wenn es sich um ein Erzeugnis der ägyptischen Kultur oder einer der Nachfolgekulturen handelt, welches auf ägyptischem Boden vor mindestens 1000 Jahren seit dem Beginn der prähistorischen Zeit entstanden ist.

Nach den insoweit im Wesentlichen übereinstimmenden Gutachten sämtlicher im Ermittlungsverfahren hinzugezogener Sachverständiger sind diese Voraussetzungen für die drei genannten Gegenstände erfüllt.

Gemäß Art. 6, 8 des ägyptischen Antikengesetzes sind diese Gegenstände öffentliches Eigentum und können nur mit schriftlicher Erlaubnis verkauft werden. Eine entsprechende Erlaubnis liegt nicht vor, wie die ägyptischen Behörden mitteilten. Auch der Verurteilte hat das Vorliegen einer solchen Erlaubnis nicht behauptet. E. M., der vor dem Verurteilten B. Besitz an den Gegenständen hatte, wurde von der Staatsanwaltschaft Bern rechtskräftig wegen Verstoß gegen das schweizerische KulturgütertransferG zu einer Geldstrafe von 100 Tagessätzen à 30 Schweizer Franken verurteilt, was den Schluss nahelegt, dass auch er eine Verkaufserlaubnis der ägyptischen Behörden nicht behauptet hat bzw. eine solche eventuelle Behauptung widerlegt wurde.

Ägypten ist daher Eigentümer der genannten drei Gegenstände.

c) Diese sind der Arabischen Republik Ägypten auch "durch die Straftat" im Sinne des § $111 \mathrm{k}$ StPO entzogen worden.

aa) Aus den Ermittlungsakten ergibt sich folgender Sachverhalt:

Die Nischenstele und der Statuenschrein waren im November 2008 noch in Ägypten und wurden dort vom Beschwerdeführer selbst fotografiert. Der Beschwerdeführer hat allerdings behauptet, die Gegenstände vor der Übergabe in Bern nie selbst gesehen zu haben, sondern im Auftrag eines A. F. bei E. M. in Bern abgeholt zu haben, um sie dem Händler J. B. zu bringen. E. M. seinerseits hatte bestritten, die Gegenstände in einem falsch deklarierten Container aus Italien eingeführt zu haben und Kontakt mit einem A. F. zu haben. E. M. wurde aber in der Schweiz durch Strafbefehl vom 15. März 2013 wegen der Einfuhr aller vier hier streitigen Gegenstände in einem falsch 
deklarierten Container aus Italien in die Schweiz rechtskräftig nach dem Schweizer KunstgütertransferG zu einer Geldstrafe verurteilt.

Bei dieser Sachlage muss davon ausgegangen werden, dass der Beschwerdeführer auf irgendeine Weise in die im Zeitraum zwischen November 2008 und Februar 2009 erfolgte unerlaubte Ausfuhr der Kulturgüter aus Ägypten verwickelt war, und zwar nach seiner eigenen Einlassung mindestens als Transporteur, möglicherweise auch als Importeur. Dass die Verfahren gegen E. M. und F. wegen Beteiligung an der Steuerhinterziehung von der Staatsanwaltschaft Lörrach nach § 170 Abs. 2 StPO eingestellt wurden, steht dem nicht entgegen. Diese Einstellungen beruhen nicht etwa auf der Annahme, die Kulturgüter seien rechtmäßig aus Ägypten exportiert worden, sondern auf der nicht geklärten Art des Zusammenwirkens zwischen dem Beschwerdeführer einerseits und den genannten anderen Personen andererseits. Die mehrfach vorgebrachte Behauptung des Beschwerdeführers, er sei davon ausgegangen, dass es sich um Fälschungen handele, ist bereits deshalb nicht überzeugend, weil er nach seiner eigenen Einlassung für die Transporte teilweise nicht unbeträchtlich bezahlt wurde und mit A. F., seiner Einlassung zufolge, schon seit vielen Jahren zusammenarbeitet. A. F. wird seinerseits seit 2003 von Ägypten wegen der unerlaubten Ausfuhr von Kulturgütern aus Ägypten mit internationalem Haftbefehl gesucht.

Die nicht deklarierte Einfuhr der genannten Gegenstände in das Bundesgebiet ist bei dieser Sachlage als Teil eines Gesamttatplans anzusehen, den der Beschwerdeführer kannte und in dem er eine bewusste Rolle spielte. Eine zollrechtliche Deklaration hätte nämlich zur zumindest vorläufigen Beschlagnahme der Kulturgüter nach §§ 8, 16 KulturgüterrückführungsG geführt.

bb) Bei Zugrundelegung dieses Sachverhalts wurden die drei genannten Gegenstände Ägypten durch die Straftat entzogen, die Gegenstand des Verfahrens war.

Zwar sind die genannten drei Gegenstände Ägypten nicht durch die materiell-rechtlich abgeurteilte Straftat nach § 370 AO selbst abhanden gekommen. Hinzu kommt, dass die - wie auch immer geartete, sich aber sowohl aus seiner Einlassung als auch aus den auf seine Tatbeteiligung als Importeur hinweisenden Indizien sicher ergebende - Beteiligung des Beschwerdeführers an der Ausfuhr der Gegenstände aus Ägypten nach deutschem Recht materiell-rechtlich nicht strafbar war. Das deutsche KulturgüterrückführungsG von 2007 enthält für eine Strafbarkeit nach § 20 dieser Vorschrift nämlich Voraussetzungen, die vorliegend - und auch in zahlreichen anderen Fällen des Verbringens von Kulturgütern aus UNESCO-Vertragsstaaten in die Bundesrepublik, vgl. Bericht der Bundesregierung zum Kulturgutschutz in Deutschland vom 15. August 2013, S. 62 f. - nicht erfüllt waren. Eine Strafbarkeit nach § 246 StGB (Unterschlagung) oder § 259 StGB (Hehlerei oder Beihilfe dazu) scheiterte an $\S \S 6,7$ StGB, da diese Taten nicht auf deutschem Boden begangen wurden und der Beschwerdeführer kein deutscher Staatsangehöriger ist.
Das Verhalten des Beschwerdeführers, das Gegenstand des umfangreichen Ermittlungsverfahrens war, stellt sich aber als einheitlicher Lebenssachverhalt im Sinne des § 264 StPO dar. Ein Teilaspekt dieses Gesamtgeschehens war auch nach deutschem Recht strafbar. Daher handelt es sich bei dem dem Verfahren zugrundeliegenden Gesamtlebenssachverhalt um „die Straftat" im Sinne des § $111 \mathrm{k} \mathrm{S.} 1$ StPO.

Dahinstehen kann bei dieser Sachlage die Streitfrage, ob es sich bei der Straftat, durch die die Gegenstände dem Verletzten im Sinne des § $111 \mathrm{k}$ StPO entzogen wurde, auch um irgendeine Straftat gehandelt haben kann, die in keiner Weise Gegenstand des Verfahrens war (so OLG Düsseldorf, NStZ 1984, 567; OLG Celle, Beschluss vom 21. Dezember 2001, 2 Ws 282/01, bei [...]) oder ob die Straftat Gegenstand des Verfahrens gewesen sein muss, in dem die Beschlagnahme erfolgte (so LR/Schäfer, StPO, § 111k, Rn. 7; SK/Wohlers, StPO, § 111k, Rn. 58 zu § 98, Meyer-Goßner, StPO, 56. Aufl., § 111k, Rn. 6 sowie Gropp, Anmerkung zu OLG Düsseldorf, NStZ 1984, 567 in NStZ 1984, 568).

cc) Die Gegenstände wurden Ägypten auch unmittelbar durch die Tat entzogen. Mit dem Unmittelbarkeitskriterium sollen die Fälle ausgeschlossen werden, in denen die beschlagnahmten Gegenstände nur noch Surrogate der ursprünglich entzogenen Gegenstände sind. Durch die Rückgabe nach § $111 \mathrm{k}$ StPO soll nämlich an den Verletzten kein Schadensersatz geleistet werden, sondern lediglich vermieden werden, dass der Staat sich durch die Rückgabe an den letzten Gewahrsamsinhaber an der Aufrechterhaltung eines rechtswidrigen Zustands beteiligt.

d) Rechte Dritter stehen der Herausgabe nicht entgegen. Der Beschwerdeführer hat nicht behauptet, weitergehende Rechte als den bloßen vorherigen Gewahrsam an den Gegenständen zu haben. Solche sind auch nicht erkennbar. Die Zollverwaltung hat der Herausgabe zugestimmt.

\section{IV.}

Bezüglich des Schnurösengefäßes sind die Voraussetzungen des § 111k StPO dagegen nicht erfüllt. Die Echtheit dieses Gefäßes wurde von den Sachverständigen unterschiedlich beurteilt. Einig waren sich die Sachverständigen darin, dass das Gefäß, wenn es echt ist, nicht aus Ägypten stammen dürfte, sondern aus einer anderen Region des Vorderen Orients. Zwar hat Ägypten ein Gutachten vorgelegt, wonach es sich bei allen vier Gegenständen um ägyptische Antiken handeln soll. Dieses ist aber nicht begründet und entkräftet daher die Zweifel an der ägyptischen Herkunft nicht, die sich aus den teilweise sehr ausführlich begründeten Gutachten ergeben, die im vorliegenden Verfahren eingeholt wurden. Diesbezüglich war die Anordnung der Herausgabe an den Verletzten daher aufzuheben und der entsprechende Antrag der Staatsanwaltschaft zurückzuweisen. (Entscheidung von der Redaktion bearbeitet.) 